\title{
AVR ST-SEGMENT ELEVATION AS PREDICTOR OF THREE-VESSEL DISEASE IN NON-ST- SEGMENT ELEVATION ACUTE CORONARY SYNDROME
}

\section{ELEVACIÓN DEL SEGMENTO ST EN AVR COMO PREDICTOR DE ENFERMEDAD CORONARIA DE 3 VASOS EN SÍNDROME CORONARIO AGUDO SIN ELEVACIÓN DEL ST}

\author{
Jonathan Cardona-Véleza ${ }^{1}$, Laura Ceballos-Naranjo².
}

Electrocardiogram (ECG) is a useful tool for the diagnosis of acute coronary syndrome (ACS). In recent years, ST-segment changes in aVR have been shown to provide valuable information about coronary anatomy and early risk stratification of $\mathrm{ACS}^{1}$. This lead gives information from the upper right portion of the heart including the right ventricle outflow tract and the basal portion of the interventricular septum ${ }^{2}$. aVR ST-segment elevation may be caused by transmural ischemia of the right ventricular outflow tract, by reciprocal changes of the left lateral leads in $\mathrm{ECG}^{1}$ and by ischemia of the basal part of the interventricular septum due to occlusion of the proximal segment of the left anterior descending artery $(\mathrm{LADA})^{2-4}$. In ACS patients this electrocardiographic finding may be accompanied by a ST-segment depression, usually in precordial leads, having an independent predictive value for global subendocardial ischemia due to occlusion of the left main trunk (LM) or three-vessels disease $(3-V D)^{1,3,5}$. Thus, this change has been associated with a higher mortality, a more extensive coronary involvement in the context of a non-ST-segment elevation ACS ${ }^{1}$ and even it correlates with a higher SYNTAX score ${ }^{3}$.

A study by Kosugue $M$ et al, found that ST-segment elevation $\geq 0.05 \mathrm{~mm}$ in lead aVR was the strongest predictor of severe LM involvement and/or 3-VD followed by a positive troponin T on admission ${ }^{5}$. Several studies have shown that ST-segment elevation in aVR compared to ST-segment depression in other leads is a better predictor of adverse outcomes in patients with non-ST-segment elevation ACS ${ }^{1}$ and anticipates LM and/or 3-VD ${ }^{3,6}$.

aVR ST-segment elevation can predict an acute occlusion of the LM, which supplies approximately $75 \%$ of the myocardial mass of the left ventricle and therefore its obstruction generates life-threatening hemodynamic deterioration and malignant arrhythmias. Hence a either percutaneous or surgical coronary revascularization should be performed as soon as possible ${ }^{2,4}$, so it is essential for doctors to identify these electrocardiographic features in order to establish a proper diagnosis and provide a timely treatment for ACS.

A 69 year-old men patient with medical record of hypertension and diabetes mellitus type 2 under medical treatment without other remarkable morbidities presented at emergency department with 1-hour history of severe uninterrupted epigastric pain radiated to precordial region associated with diaphoresis, asthenia, nauseas and a Killip-Kimball class-2. Troponin I was positive and electrocardiogram on admission showed an aVR ST-segment elevation accompanied of a ST-segment depression in leads II, III and aVF (Figure 1).

\footnotetext{
1 Residente Medicina Interna, Universidad de Antioquia. Hospital Universitario San Vicente Fundación. Medellín, Colombia. 


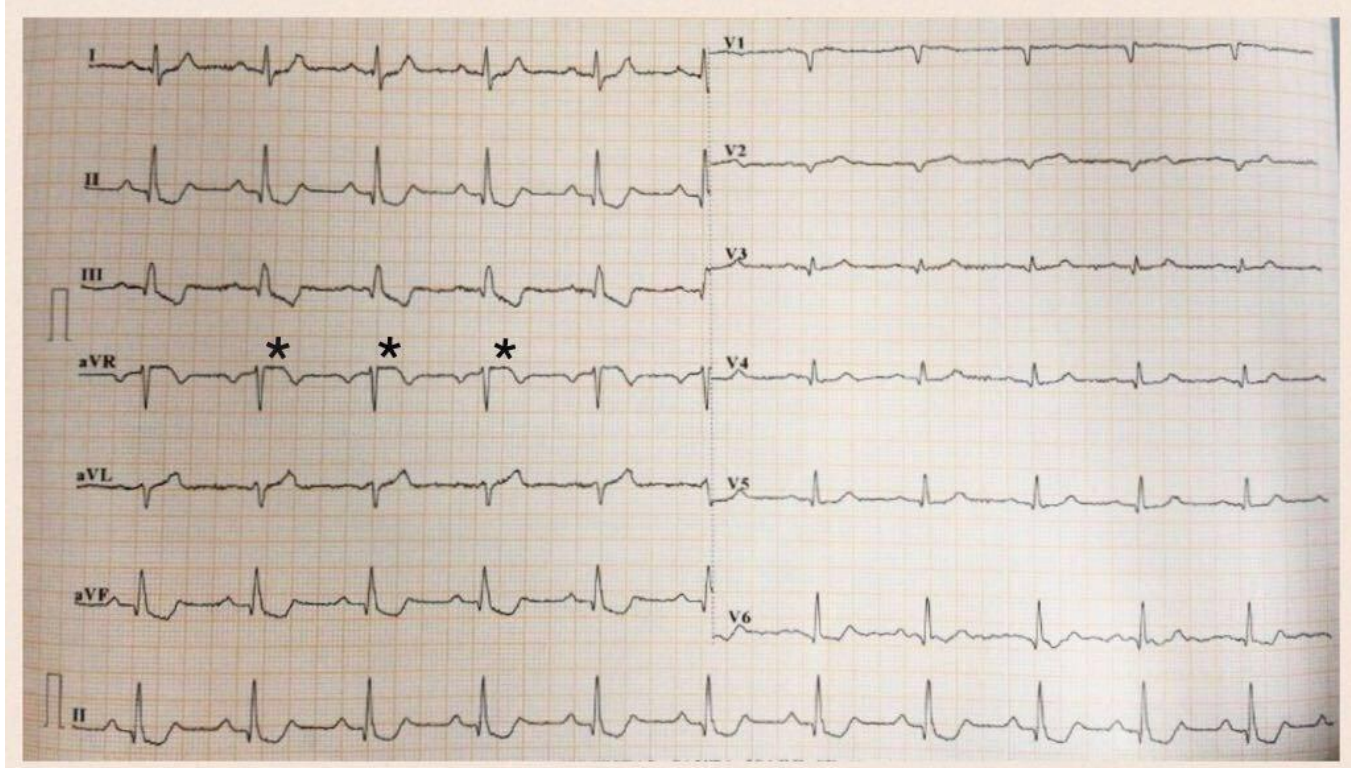

Figure 1. Electrocardiogram showing aVR ST-segment elevation.

A coronary angiography was performed immediately, which revealed $95 \%$ stenosis at the proximal-right coronary artery (RCA), diffuse involvement of proximal-mid portion of LADA and a $99 \%$ critical occlusion at left circumflex artery (LCA). Percutaneous coronary intervention with stent placement was done in three vessels involved, using two drug-eluting stent in LAD, another one on RCA and a bare metal stent on LCA, restoring completely the flow and getting a TIMI 3 flow (Figure 2).
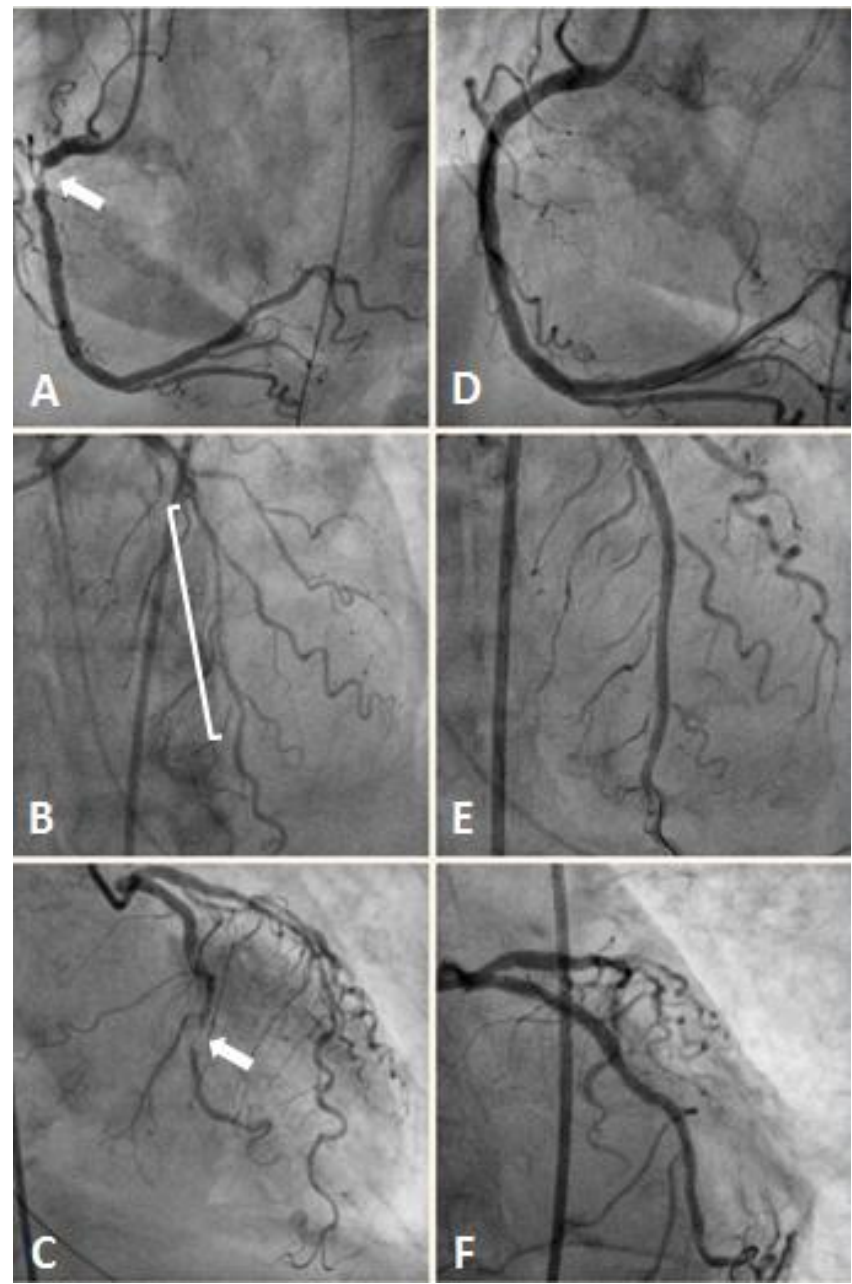

Figure 2. Coronary angiography revealing $95 \%$ stenosis at the proximal-right coronary artery (A), diffuse involvement of left anterior descending artery

(B) and $99 \%$ critical occlusion on mid-left circumflex artery (C). (D), (E) and (F) panels show coronary anatomy after percutaneous coronary intervention and stent placement. 


\section{Bibliography}

1 Yan AT, Yan RT, Kennelly BM, et al; GRACE investigators. Relationship of ST elevation in lead aVR with angiographic findings and outcome in non-ST elevation acute coronary syndromes. Am Heart J 2007; 154: 71-8.

2. Tamura A. Significance of lead aVR in acute coronary syndrome. World J Cardiol. 2014;6(7):630-7.

3. Cerit L. Chicken or the egg: ST elevation in lead aVR or SYNTAX score. Cardiovasc J Afr. 2016;27:1-4.

4. Kosuge M, Kimura K, Ishikawa $T$, et al. Predictors of left main or three-vessel disease in patients who have acute coronary syndromes with non-ST-segment elevation. Am J Cardiol 2005; 95: 1366-9.

5. Kosuge M, Ebina T, Hibi K, et al. An early and simple predictor of severe left main and/or three-vessel disease in patients with non-ST-segment elevation acute coronary syndrome. Am J Cardiol 2011; 107: 495-500.

6. Misumida N, Kobayashi A, Fox JT, at al. Predictive Value of ST-Segment Elevation in Lead aVR for Left Main and/or Three-Vessel Disease in Non-ST-Segment Elevation Myocardial Infarction. Ann Noninvasive Electrocardiol. 2016;21(1):91-7. 